\title{
Paeoniflorin Effect of Schwann Cell-Derived Exosomes Ameliorates Dorsal Root Ganglion Neurons Apoptosis through IRE1 $\alpha$ Pathway
}

\author{
Yanbo Zhu $\mathbb{D}^{1,2}$ Shuo Han, ${ }^{1,2}$ Xiao Li, ${ }^{1,2}$ Yingying Gao, ${ }^{1,2}$ Jiayue Zhu, ${ }^{1,2}$ Xinwei Yang, \\ and Liping $\mathrm{Xu}\left(\mathbb{C}^{1,2}\right.$ \\ ${ }^{1}$ School of Traditional Chinese Medicine, Capital Medical University, Beijing, China \\ ${ }^{2}$ Beijing Key Lab of TCM Collateral Disease Theory Research, Capital Medical University, Beijing, China \\ Correspondence should be addressed to Liping Xu; xulp@ccmu.edu.cn
}

Received 30 June 2021; Accepted 20 August 2021; Published 27 September 2021

Academic Editor: Rajeev K Singla

Copyright (c) 2021 Yanbo Zhu et al. This is an open access article distributed under the Creative Commons Attribution License, which permits unrestricted use, distribution, and reproduction in any medium, provided the original work is properly cited.

\begin{abstract}
Background. Diabetic peripheral neuropathy (DPN) is a common complication of diabetes but its pathogenesis is not fully clarified. Endoplasmic reticulum (ER) stress has been confirmed to be involved in the development of DPN. Dorsal root ganglion neuron (DRGn) is the target cell of DPN injure in the peripheral neurons system. Schwann cell (SCs)-derived exosomes (SCEXOs) can carry IRE1 $\alpha$ signal transduction factors in ER stress to DRGn. The aim of this study is to investigate the effect of SCEXOs treated with paeoniflorin (PF) on DRGn stimulated by high glucose. Methods. SCs were divided into Control group (Control), $150 \mathrm{mM}$ glucose group (HG), high osmotic pressure group (HOP), and low, middle, and high dose PF group (PF1, PF10, and PF100). Exosomes were obtained from SCs by ultracentrifugation and identified according to marker proteins, including CD63, Alix, Hsp70, and TSG101. ER stress initiating factor GRP78, the IRE1 $\alpha$ pathway information transmission factor IRE1 $\alpha$, and the phosphorylation level of IRE1 $\alpha$ were detected by Western blot, DRGn is divided into Control group (Control), $50 \mathrm{mM}$ glucose group + Control exosomes group (HG + EXOs Control), $50 \mathrm{mM}$ glucose group (HG), and $50 \mathrm{mM}$ glucose group + administration exosomes group (HG + EXOs PF1, HG + EXOs PF10, and HG + EXOs PF100); ER morphology of primary DRGn was observed by using the transmission electron microscope, the level of DRGn apoptosis was analyzed by TUNEL, and the downstream proteins of ER stress including CHOP, XBP1S, JNK, and p-JNK in DRG and the expression of apoptosis-related proteins Bcl-2, Bax, Caspase-3, and Caspase-12 were measured by Western blot. Results. Compared with the exosomes in the HG group, the exosomes after the intervention of PF can significantly reduce the expression of GRP78, IRE1 $\alpha$, and the phosphorylation level of IRE1 $\alpha(P<0.05)$; compared with the DRGn in the HG group, the SC-EXOs treated with PF could regulate the expression of proteins downstream of IRE1 $\alpha$ pathway in ER stress $(P<0.05$ or $P<0.01)$, improve the morphological integrity of ER, and reduce apoptosis in DRGn $(P<0.05$ or $P<0.01)$. Conclusion. PF regulates the information of ER stress carried by SCEXOs and further affects downstream of IRE1 $\alpha$ pathway in DRGn, thus reducing ER stress-induced apoptosis. PF can interfere with DPN through affecting information communication carried by EXOs between SCs and DRGn.
\end{abstract}

\section{Introduction}

Diabetic peripheral neuropathy (DPN) is one of the most common long-term complications of diabetes, with a prevalence of over $50 \%$, and is characterized by sensory and motor neuron damage $[1,2]$. The pathogenesis of DPN is complex, including microvascular ischemia, polyol pathway, oxidative stress, and endoplasmic reticulum stress (ER stress) [3]. Our previous studies have shown that apoptosis induced by ER stress is one of the important mechanisms of the pathogenesis of DPN [4-6]. Endoplasmic reticulum (ER) is an important organelle for protein synthesis, folding and modification, lipid synthesis, and calcium storage. ER stress is caused by the accumulation of unfolded or misfolded proteins, interruption of lipid synthesis, or depletion of calcium storage. High glucose stimulation can cause ER 
stress in cells, and the cells respond to ER stress through unfolded protein response (UPR) to maintain ER homeostasis. UPR mainly contains PERK, ATF6, and IRE $1 \alpha$ signal pathways [7]. IRE1 $\alpha$ pathway is the most conservative branch of UPR in evolution, which can reduce the protein folding load and increase the ER proteins folding ability [8].

Dorsal root ganglion (DRG) is one kind of cell bodies, and the susceptibility of dorsal root ganglion neurons (DRGn) to high glucose concentration stress in vivo and in vitro is involved in the occurrence and development of diabetic neuropathy. At the same time, ER stress can also cause abnormal DRGn ion channel function, gene expression, transcription regulation, metabolism, and protein folding [9].

As a kind of lipid bilayer membrane vesicles involved in cell-to-cell communication, exosomes play a key role in intracellular communication [10]. Exosomes are wrapped in bilayer membranes to protect their genetic material. In this way, exosomes can be used as stable and effective carriers to carry specific goods such as proteins, lipids, and genetic materials, so they can be used as a promising tool to transport anti-infective goods to target tissues or organs [11]. Extracellular pathway is an effective way to regulate apoptosis, angiogenesis, and target cell inflammation [12]. The SC-EXOs can significantly promote axonal growth and regeneration both in vitro and in vivo $[13,14]$. Axon regeneration is an important process for functional recovery after being stimulated by high glucose $[15,16]$. Therefore, SC-EXOs may be used as carriers to participate in the regulation of DRGn axon regeneration and growth and promote the repair of peripheral nerves after injury [17]. The study showed that the secretion of exosomes would increase under ER stress, but in the IRE1 $\alpha$-deficient cells, the secretion of exosomes did not increase. This indicated that the IRE1 $\alpha$ pathway was involved in the release of exosomes [18]. At the same time, with ER stress, the endoribonuclease activity activated by IRE1 $\alpha$ will cleave XBP1 (X-box binding protein 1) mRNA which could play the role of transcription factor. The IRE1 $\alpha$-XBP1 pathway was involved in insulin resistance and dyslipidemia. It has been previously demonstrated that IRE1 $\alpha$ was essential for the release of exosomes from palmitate-treated liver cells $[19,20]$. However, the role of exosomes stimulated by IRE1a and the regulation of the IREla pathway are still unclear.

Paeoniflorin (PF) is a monoterpene glycoside isolated from Ranunculaceae plant Paeonia lactiflora, which acts as antioxidant and anti-inflammatory and performs vasodilatation and other biological activities [21]. PF can block the PERK pathway by inhibiting the expression of GRP78 and finally reduce ER stress [22]. At the same time, the antiinflammatory effect of PF is achieved by inhibiting the phosphorylation level of IRE1 $\alpha[23,24]$. Therefore, this article studies the effect of PF on SC-EXOs in IRE1 $\alpha$ signaling pathway of DRGn.

\section{Materials and Methods}

2.1. Primary DRGn Extraction and SCs Culture. DRGn was prepared from SD neonatal rats regardless of gender (3-5 days old, Charles River Experimental Animal Technology
Co., Ltd.). The DRG is taken from the intervertebral foramen of both vertebrae. The tail of the DRG is cut off, and its head is retained. The head is cut into pieces and incubated in $4 \mathrm{~mL}$ trypsin with $1 \%$ collagenase for $1-2$ hours at $37^{\circ} \mathrm{C}$. Dissociated cells were seeded in Petri dish coated with poly-Llysine (PLL, Sigma-Aldrich), incubated for 7 days, and the medium is replaced every 2-3 days $[25,26]$.

SCs line was cultured in DMEM complete medium containing $10 \%$ fetal bovine serum (FBS) and $1 \%$ penicillinstreptomycin. The incubator was kept at $37^{\circ} \mathrm{C}$ and $5 \% \mathrm{CO}_{2}$. They were divided into Control group $(25 \mathrm{mmol} / \mathrm{L}$ glucose DMEM + 10\% FBS, Control), high glucose group $(150 \mathrm{mmol} / \mathrm{L}$ glucose DMEM $+10 \%$ FBS, HG), high osmotic pressure group $(25 \mathrm{mmol} / \mathrm{L}$ glucose DMEM + $44.4 \mathrm{mmol} / \mathrm{L}$ Mannitol + 10\% FBS, HOP), PF low dose group $(150 \mathrm{mmol} / \mathrm{L}$ glucose DMEM $+10 \% \mathrm{FBS}+1 \mu \mathrm{M} \mathrm{PF}$, $\mathrm{PF} 1$ ), $\mathrm{PF}$ medium dose group (150 mmol/L glucose DMEM $+10 \% \mathrm{FBS}+10 \mu \mathrm{M} \mathrm{PF}, \mathrm{PF} 10)$, and PF high dose group $(150 \mathrm{mmol} / \mathrm{L}$ glucose $\mathrm{DMEM}+10 \% \mathrm{FBS}+100 \mu \mathrm{M}$ PF, PF100). After 24 hours of modeling, the supernatant was collected to extract exosomes.

2.2. Exosomes Extraction. SC-EXOs were extracted by ultracentrifugation from the culture supernatants. SC-EXOs were extracted by ultracentrifugation from the culture supernatants. The supernatants were collected and sequentially centrifuged firstly at $300 \times \mathrm{g}$ for $10 \mathrm{~min}$, then the supernatants were collected and secondly at $2,000 \times \mathrm{g}$ for 20 $\mathrm{min}$. Then again they were collected; next $30 \mathrm{~min}$ with $10,000 \times \mathrm{g}$. The supernatant was transferred into the $8.9 \mathrm{~mL}$ centrifugal tube (Beckman, USA) to ultracentrifugation at $100,000 \times \mathrm{g}$ for $70 \mathrm{~min}$ twice at $4^{\circ} \mathrm{C}$ to get the exosomes. Our extraction method was the same as described in the previous study [27]. After sucking out all the supernatant, the precipitate was dissolved in $200 \mu \mathrm{L}$ PBS and collected in $1.5 \mathrm{~mL}$ centrifuge tube for follow-up experiments.

2.3. Cell CCK8 Assay. 100 uL SCs suspension was inoculated in each well of 96-well plate, and Control group $(25 \mathrm{mmol} / \mathrm{L}$ glucose DMEM + 10\% FBS), HG group ( $150 \mathrm{mmol} / \mathrm{L}$ glucose DMEM + 10\% FBS, HG), and HOP group ( $25 \mathrm{mmol} / \mathrm{L}$ glucose DMEM + $44.4 \mathrm{mmol} / \mathrm{L}$ Mannitol $+10 \% \mathrm{FBS}$ ) were set up. Six multiple holes were set in each group. After 24 hours, $10 \mu \mathrm{l}$ CCK8 solution was added to each hole. The culture plate was incubated in the incubator for 3 hours. The absorbance value was measured by enzyme labeling instrument at $450 \mathrm{~nm}$. Cytoactive rate $(\%)=[(\mathrm{As}-\mathrm{Ab}) /$ $(\mathrm{Ac}-\mathrm{Ab})] \times 100 \%$, where As is HG or HOP group absorbance; $\mathrm{Ac}$ is Control group absorbance; and $\mathrm{Ab}$ is Blank hole absorbance.

2.4. Confocal Laser Microscopy. The primary DRGn was identified, and the SC-EXOs internalized by DRGn were observed with immunofluorescence.

DRGn identification: DRG was extracted and inoculated into cell climbing tablets and cultured for 7 days. DRGn was stained with NF-H polyclonal antibody (18934-1-AP, 
Proteintech, NF200) for specific immunofluorescence staining, and the morphology of DRGn was observed by using laser confocal microscope.

DRGn internalized SC-EXOs: DRGn was stained by immunofluorescence with NF-H polyclonal. The exosomes were labeled with PKH26 (MINI26-1KT, Sigma) and cocultured with DRGn for 24 hours. The situation of SC-EXOs internalized by DRGn was observed by immunofluorescence.

2.5. Transmission Electron Microscopy. The primary DRGn was inoculated on a six-well culture plate and cultured for 7 days. DRGn collected by centrifugation was fixed with glutaraldehyde for 2 hours, and the samples were rinsed 3 times with phosphate buffer. It is taken to Core Facility Center of Capital Medical University. The ER in DRGn was observed by using the HT7700 transmission electron microscope (Hitachi, Japan). The clear ER image was finally obtained by using the CCD camera and the microscope host.

2.6. Western Blot. The treated DRGn and SC-EXOs were collected. Cellular protein was extracted by RIPA lysis buffer. The equal proteins were separated on an SDS-PAGE, and then they are transferred to the PVDF membrane. The membrane was blocked with 5\% nonfat dry milk for 1 hour. The PVDF membrane was incubated overnight at $4^{\circ} \mathrm{C}$ with primary antibodies, including Alix (1:2000, ab275377, Abcam), Hsp70 (1:2000, ab2787, Abcam), CD63 (1:2000, ab134045, Abcam), TSG101 (1:2000, ab125011, Abcam), GRP78 (1:2000, ab108613, Abcam), IRE1 $\alpha$ (1:500, sc390960, Santa cruz), p-IRE1 $\alpha$ (1:2000, ab124945, Abcam), CHOP (1:500, sc-46661, Santa cruz), JNK (1:2000, ab208035, Abcam), p-JNK (1:2000, ab124956, Abcam), XBP1 (1 : 500, sc-8015, Santa cruz), Bcl-2 (1:2000, ab182858, Abcam), Bax (1:2000, ab32503, Abcam), Caspase-3 (1:2000, ab32351, Abcam), Caspase-12 (1:2000, ab62484, Abcam), and $\beta$-actin $(1: 2000$, ab8226, Abcam). The membranes were incubated with the appropriate horseradish peroxidaseconjugated secondary antibodies. The specific bands were observed by using the hypersensitive ECL chemiluminescence kit (New Cell \& Molecular Biotech Co., Ltd, P10200).

2.7. TUNEL Assay. DRGn was cultured in groups on the cell climbing slice; the cells were fixed by $4 \%$ paraformaldehyde, $0.1 \%$ TritonX-100 permeated through the membrane, dripped with TUNEL reaction solution (G1501-50T, Servicebio), and incubated at $37^{\circ} \mathrm{C}$ for $2 \mathrm{~h}$. The nuclei were stained with DAPI and then sealed with antifluorescence quenchant. Under the 880 Airyscan laser confocal microscope (Zeiss, Germany), 8 visual fields were randomly selected in each group, the number of apoptotic cells and total cells were counted, and the percentage of apoptosis was calculated.

2.8. Statistical Analysis. The software used to process data includes GraphPad Prism8.0 and Image J. The results are shown as mean \pm standard error of mean (SEM). Differences were analyzed by one-way ANOVA followed by Tukey's multiple comparisons test. Student's unpaired $t$-test was used to analyze data between 2 groups. $P<0.05$ was considered statistically significant.

\section{Result}

3.1. The Cytoactivity of SCs Was Not Affected by High Osmotic Pressure. Compared with Control group, the cytoactivity in HG group was significantly decreased $(P<0.01)$, while the HOP group had no significant difference (Figure 1). This experiment confirmed that high osmotic pressure had no effect on cell cytoactivity. The changes in SCs and SC-EXOs were caused by high glucoses concentration, not osmotic pressure.

3.2. Identification of SC-EXOs Incubated in High Glucose. Figure 2 shows the results of Western blot. The exosomes specific proteins Alix, Hsp70, CD63, and TSG101 were detected. They were expressed in the exosomes of each group. The expression of the marker protein indicated the presence of exosomal components which proved that SCs can secrete exosomes, and using the ultracentrifugation to extract exosomes was reliable.

3.3. Identification of Primary DRGn and Internalization of SCEXOs. Identification of primary DRGn: in order to verify whether the primary DRGn could be grown adherently after extracting the DRG by our method, we used the currently recognized DRGn specific antibody NF200 for identification. Cultured for 7 days, the immunofluorescence results showed that the adherent cells could appear green fluorescence, indicating that the adherent cells could bind to the specific antibody NF200, and the cells also showed the characteristics of DRGn: the whole cell was triangular and had slender axons (Figure 3(a)). This proved that our extraction method is correct, and the cell was primary DRGn.

The DRGn showed green fluorescence, and the nucleus showed blue fluorescence by using the laser confocal microscope. The exosomes showed red fluorescence. After merging, the red exosomes were located in the cytoplasm of green DRGn cells and distributed around the blue DRGn nucleus (Figure 3(b)). This proved that DRGn can internalize exosomes.

3.4. PF Regulated GRP78 and IRE1 $\alpha$ in SC-EXOs Incubated in High Glucose. Compared with the Control group, the expression of GRP78 in the HG group increased $(P<0.05)$; compared with the HG group, the expression of the PF1 group decreased $(P<0.05$; Figure $4(\mathrm{~b}))$. Compared with the Control group, the expression of IRE $1 \alpha$ and p-IRE $1 \alpha$ in the HG group increased $(P<0.01)$; compared with the $\mathrm{HG}$ group, the PF10 group decreased $(P<0.05$; Figures 4 (c) and $4(d))$. Thus, it could be seen that high glucose can lead to ER stress, PF can play a role in anti-ER stress, and exosomes participate in the information transmission of IRE $1 \alpha$ pathway. 


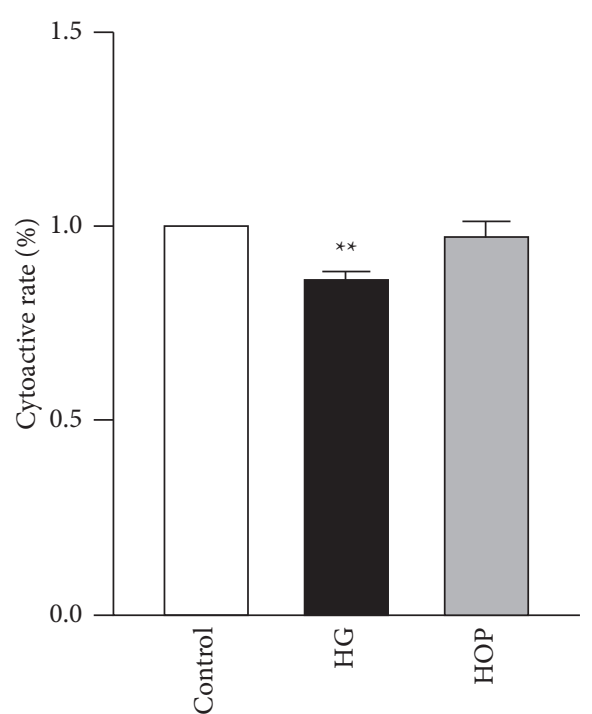

FIgURe 1: Determination of SCs high glucose and high osmotic pressure cell cytoactivity by CCK $8{ }^{\star \star} P<0.01$ vs. Control, $n=6$ for each group.

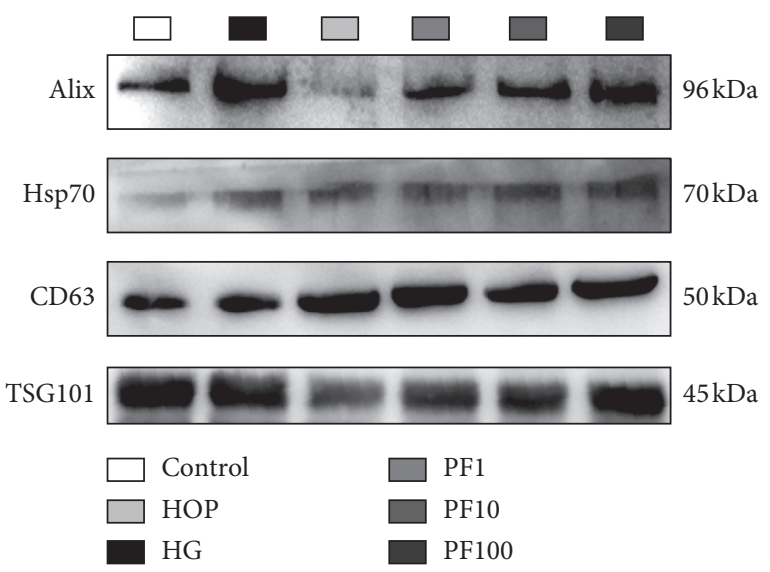

FIgURE 2: Exosomes marker protein of SC-EXOs. Western blot showed specific markers Alix, CD9, Hsp70, CD63, and TSG101 in exosomes.

3.5. Effect of PF Intervention of SC-EXOs on the Downstream

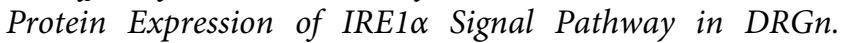
CHOP, XBP1s, C-Jun N-terminal kinase (JNK), and p-JNK are regarded as the downstream indexes of ER stress IRE1 $\alpha$ pathway $[28,29]$. We verify their expression by Western blot. Compared with the Control group, the expression of $\mathrm{CHOP}$, JNK, and p-JNK increased, and the expression of XBP1s decreased in the HG group $(P<0.05$ or $P<0.01)$. Compared with the HG group, the expression of CHOP, JNK, and p-JNK significantly decreased and the expression of XBP1s increased in the exosomes intervention group $(P<0.05$ or $P<0.01$; Figures $5(\mathrm{~b})-5(\mathrm{e}))$. This proved that the exosomes after the intervention of PF can reduce the ER stress.

3.6. Effect of PF Intervention of SC-EXOs on the Morphological Integrity of ER in DRGn. We can see that the shape of ER in the Control group is uniform (Figure 6(a)). After 24 hours of
$50 \mathrm{mM}$ glucose intervention, the morphology of ER was partially enlarged, broken, and fragmented (Figure 6(c)). In the $50 \mathrm{mM}$ glucose + EXOs PF group, the structure of ER tended to be intact (Figures 6(d) and 6(e)). This can explain that the PF interferes with SC-EXOs to maintain the intact morphology of ER. The morphology of ER in SC-EXOs PF100 and SC-EXOs Control group has improved but the effect was not obvious (Figures 6(b) and 6(e)).

\subsection{PF Interfered with SC-EXOs to Reduce Apoptosis of DRGn}

3.7.1. PF Interfered with SC-EXOs on Apoptosis of DRGn in High Glucose Concentration by TUNEL Kit. The TUNEL kit method is currently recognized as one of the methods to detect the level of apoptosis. Compared with the Control group, a large number of green fluorescence appeared in the HG group (Figure $7(a)$ ), and the level of apoptosis increased in the HG group $(P<0.01$; Figure $7(b))$. Compared with the HG group, different doses of PF interfere with the SC-EXOs to reduce apoptosis of DRGn $(P<0.01$; Figure $7(\mathrm{~b}))$. Compared with the $\mathrm{HG}+\mathrm{EXOs} \mathrm{PF} 1$ group, the apoptosis rate of DRGn in the HG+EXOs PF10 group and $\mathrm{HG}+$ EXOs PF100 group was significantly decreased $(P<0.01)$. The intervention effect of PF with doses of $10 \mu \mathrm{M}$ and $100 \mu \mathrm{M}$ was the best.

3.7.2. PF Interfered with SC-EXOs on DRGn Apoptotic Protein in High Glucose Concentration. In addition to using the TUNEL kit to detect the degree of apoptosis in DRGn, we also verified the expression of anti-apoptotic protein $\mathrm{Bcl}-2$ and pro-apoptotic protein Bax and Caspase family proteins Caspase- 12 and Caspase- 3 by Western blot. Compared with the Control group, the expression of Bax, Caspase-3, and Caspase-12 was significantly increased and the expression of Bcl-2 was decreased in the HG group $(P<0.05$ or $P<0.01)$; compared with the HG group, the expression of Bax, Caspase-3, and Caspase-12 was significantly decreased and the expression of Bcl-2 was significantly increased in the $\mathrm{HG}+$ EXOs PF group $(P<0.05$ or $P<0.01$; Figures $8(\mathrm{~b})-$ 8(e)). Western blot showed that EXOs interfered with PF could inhibit the expression of apoptotic protein in DRGn, which was consistent with the results of the previous TUNEL kit. It was more confirmed that EXOs after the intervention of $\mathrm{PF}$ can reduce the apoptosis induced by high glucose.

\section{Discussion}

In diabetic peripheral neuropathy, the research group has found that exosomes could play the role of antioxidative stress through information communication and then improve DPN. There was a variety of pathogenesis of DPN. The studies have shown that exosomes can improve DPN by regulating the communication between SCs and DRGn. ER stress is one of the pathogenesis of DPN [30]. PF can improve the apoptosis of SCs in high glucose concentration by reducing ER stress and has the potential to interfere with DPN [6]. However, can PF interfere with DPN by improving the communication between SCs and DRGn? What is the 

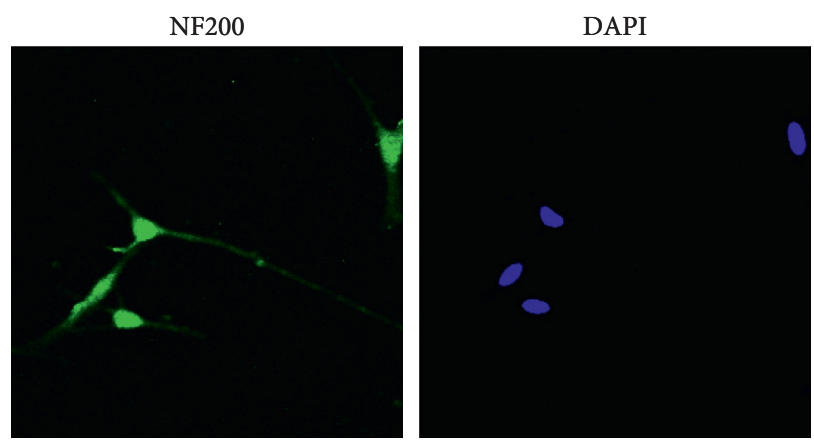

(a)
NF200

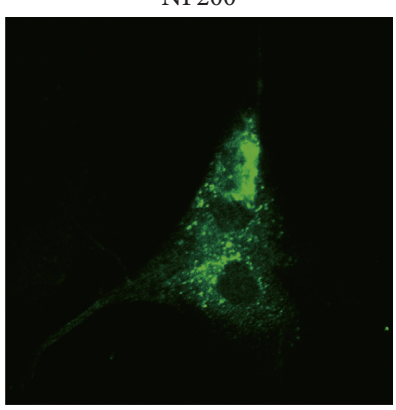

PKH26

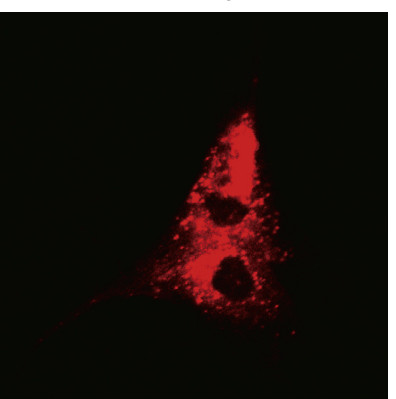

DAPI

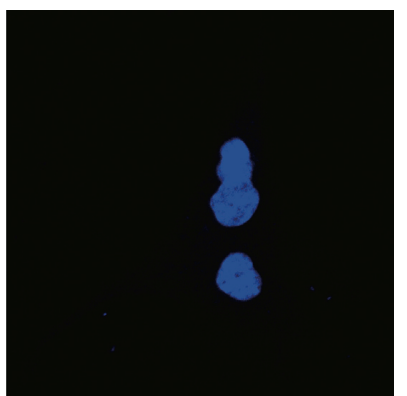

Merge

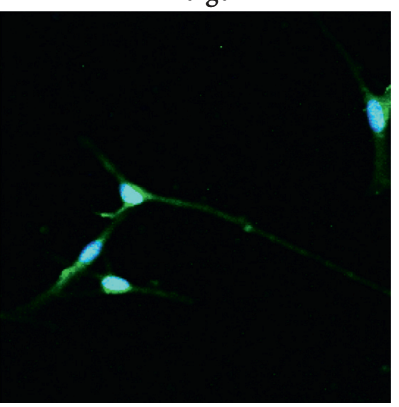

(b)

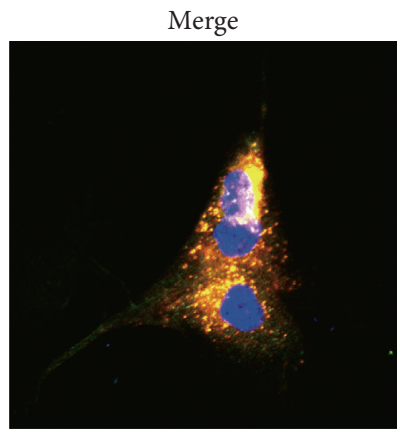

Merge

FIGURE 3: Identification of the DRGn and the internalization of SC-EXOs. (a) NF200 specific identification of DRGn $(\times 200)$, scale bar, $100 \mu \mathrm{m}$. (b) DRGn internalized SC-EXOs $(\times 400)$, scale bar, $50 \mu \mathrm{m}$.

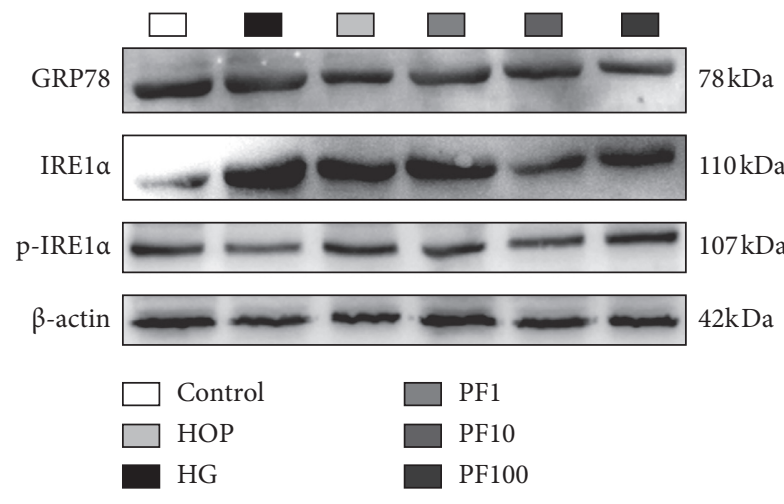

(a)

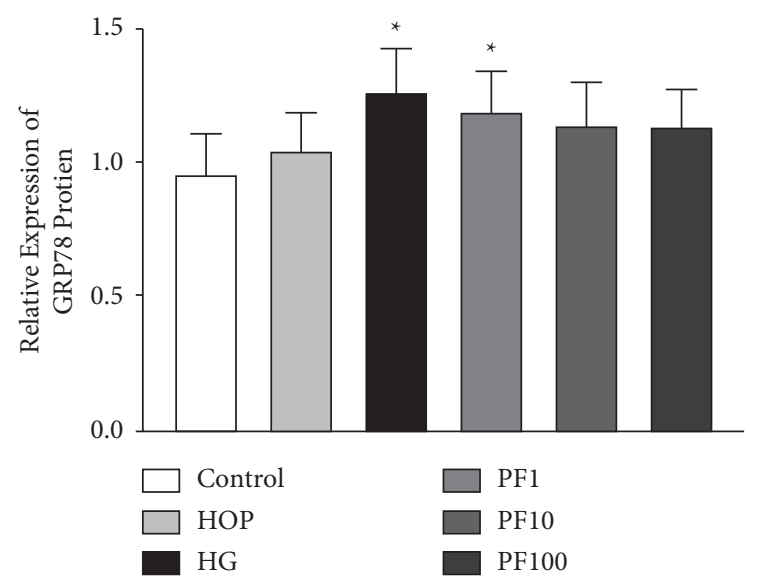

(b)

FIgURE 4: Continued. 


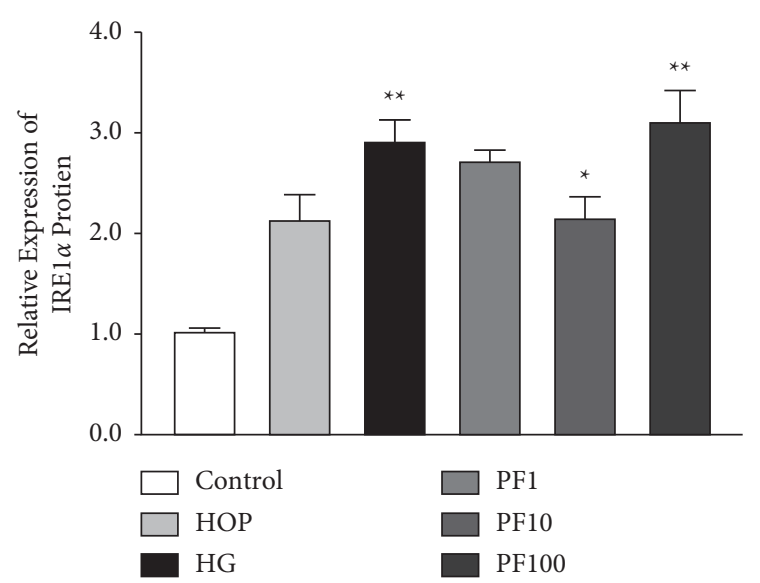

(c)

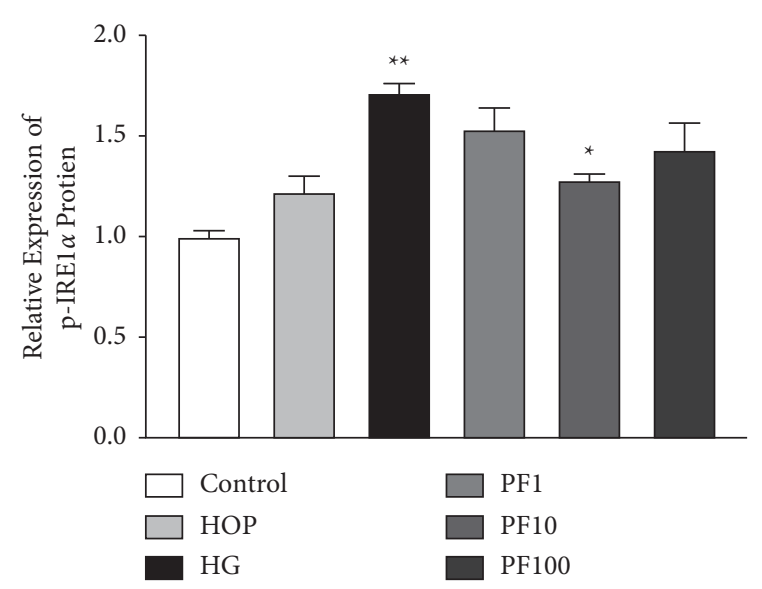

(d)

FIgURE 4: PF interfered with SC-EXOs carrying ER stress information. (a) The expression of ER stress initiating factors in exosomes. The protein expression level for (b) GRP78, (c) IRE1 $\alpha$, and (d) p-IRE1 $\alpha$ was determined by Western blotting, $n=4$ for each group. The results are expressed as the mean \pm standard error.
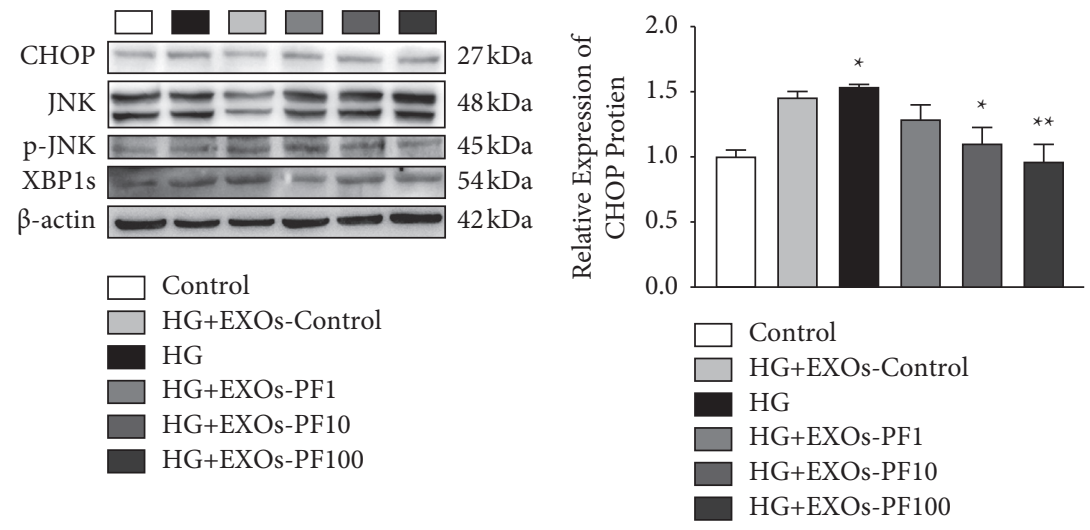

(a)

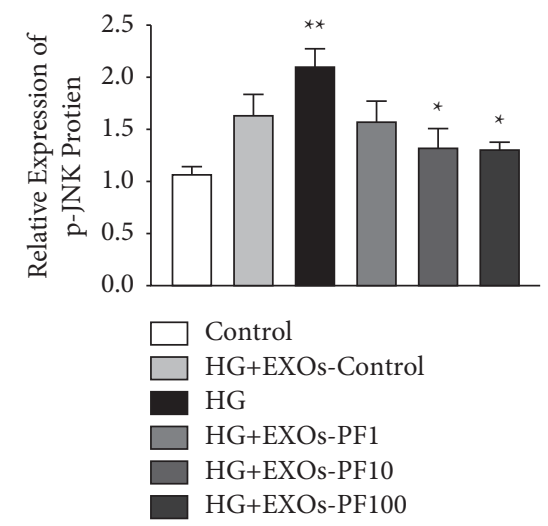

(d)

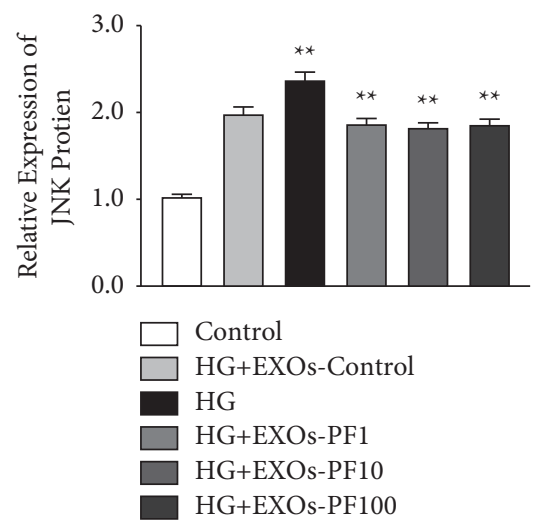

(c)

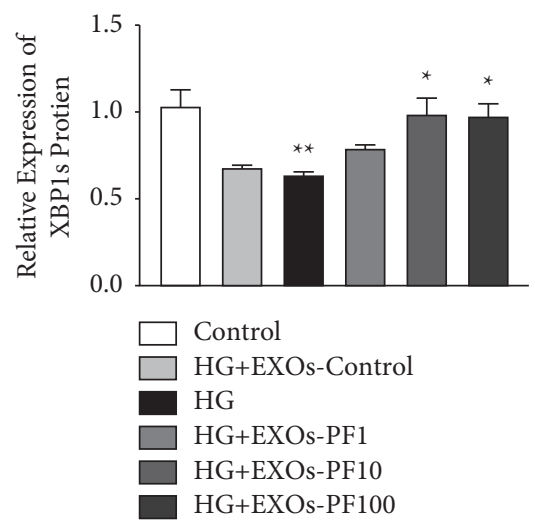

(e)

FIgUre 5: The effect of PF interfered with SC-EXOs on the downstream proteins of ER stress in DRGn. (a) The expression of ER stress downstream protein in DRGn. The expression level of (b) CHOP, (c) JNK, (d) p-JNK, and (e) XBP1s was determined by Western blotting, $n=4$ for each group. The results are expressed as the mean \pm standard error. 


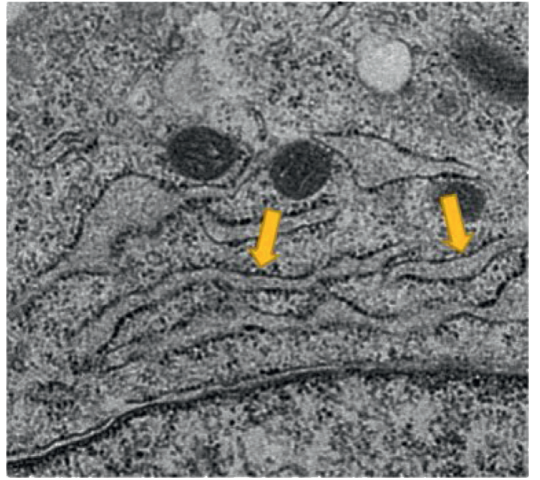

(a)

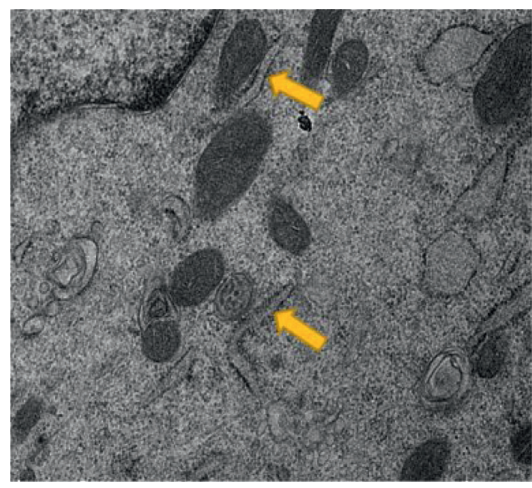

(d)

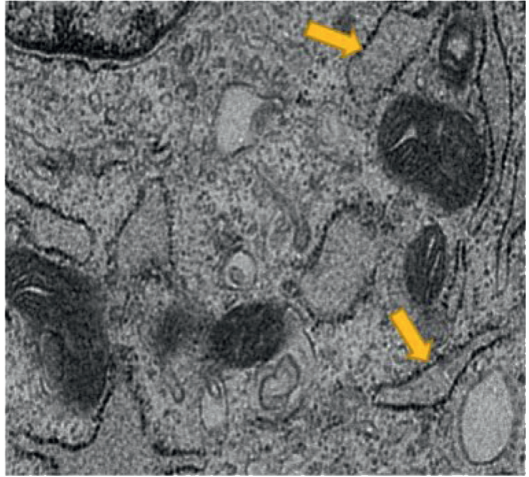

(b)

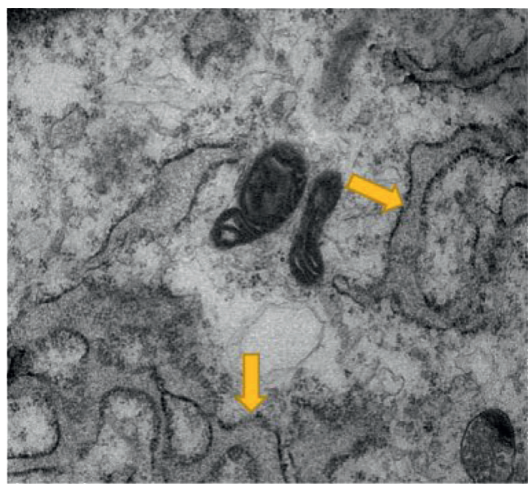

(e)

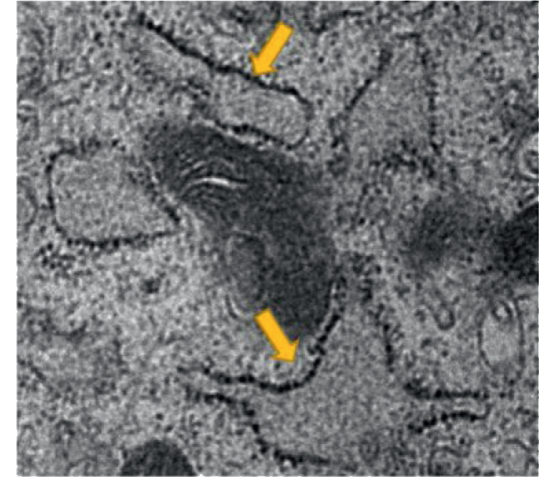

(c)

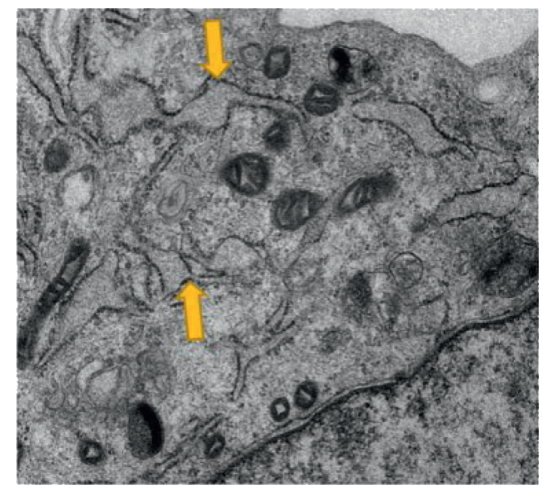

(f)

FIgURE 6: Ultrastructural observation of ER (7,000x). The yellow arrow refers to the ER. (a-f) The Control group, HG + EXOs Control group, HG group, HG + EXOs PF1 group, HG + EXOs PF10 group, and HG + EXOs PF100 group.

specific way of action? These have not been fully clarified at present. Our study confirmed that PF can reduce the apoptosis of DRGn by regulating the signal proteins related to ER stress in SC-EXOs.

In this study, ER stress was used as a breakthrough point to further explain the role of SC-EXOs. We used an in vitro model to verify this hypothesis. The results of this study are as follows: (1) SC-EXOs can carry ER stress initiating factor and IRE1 $\alpha$ pathway initiating factor; (2) the hyperosmotic condition does not affect the activity of SCs and the SCEXOs; (3) DRGn can internalize the SC-EXOs and use the exosomes communication mechanism to transmit information; (4) high glucose can aggravate the ER stress and change the morphology of ER in DRGn; and (5) SC-EXOs under the intervention of PF can reduce the apoptosis of DRGn in high concentration.

DPN as a disease of the peripheral nervous system, high glucose stimulation can cause damage to SCs and DRGn axons [31]. SC-EXOs can promote the activity of nerve cells [32]. At the same time, the SC-EXOs also can significantly promote axonal growth and axonal regeneration. Axon regeneration is an important process of functional recovery after peripheral nervous system injury [33]. Therefore, the SC-EXOs may be involved in the axonal regeneration and growth regulation of DRGn and promote the repair of peripheral nerve injury. Then, the molecular mechanism of SC-EXOs is explained to promote DRGn axonal growth, and thus ER stress is inhibited to reduce cell apoptosis; this is the core problem solved in this study.

We previously found that hyperglycemia can cause axonal damage in DRGn and trigger DPN. The degeneration of distal sensory axons in DRGn is the main cause of DPN $[34,35]$. SCs can interact with the axons of DRGn, and SCs in the normal concentration can secrete exosomes to promote the proliferation of injured DRGn [36]. SC-EXOs were internalized by DRGn axons, and miR-27a in exosomes promoted neurite growth of diabetic DRGn [30]. Our results showed that DRGn could internalize SC-EXOs, and exosomes were involved in the recovery of damaged DRGn. However, our results showed that the exosomes under the intervention of PF significantly alleviated the ER stress and apoptosis of DRGn. SC-EXOs under normal conditions can have a therapeutic effect on DRGn stimulated by high glucose, but the results are not significantly different. Therefore, it can be shown that PF intervention can help exosomes carry IRE1 $\alpha$ pathway information to inhibit apoptosis.

It has been proved that the condition of high glucose concentration can increase the number of exosomes and interfere with the biological activity of exosomes [37]. SCs with axonal damage can also release a large number of exosomes [38]. Therefore, it is proved that high glucose concentration can affect the exosomes, and we have previously proved that high glucose concentration can increase 

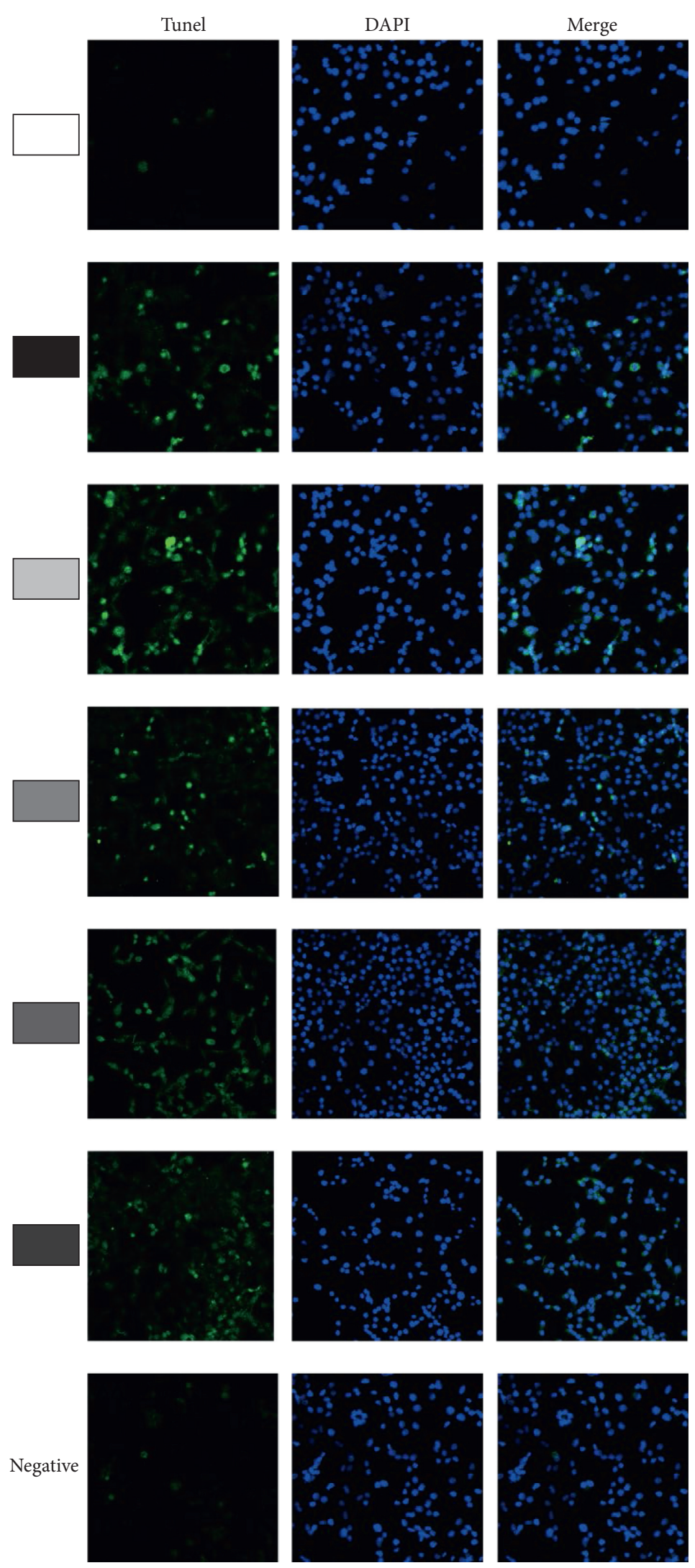

(a)

FIgURE 7: Continued. 


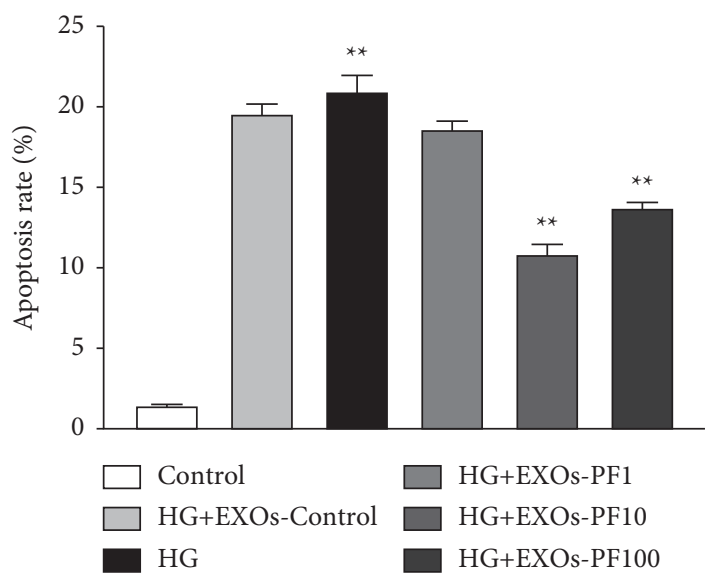

(b)

Figure 7: The apoptosis level of DRGn was detected by immunofluorescence $(\times 200)$ dcale bar, $100 \mu \mathrm{m}$. The green fluorescence was the apoptotic cells stained by TUNEL kit, and the blue fluorescence was the nucleus. Blue-green overlap was the apoptotic cells. $n=6$ for each group.

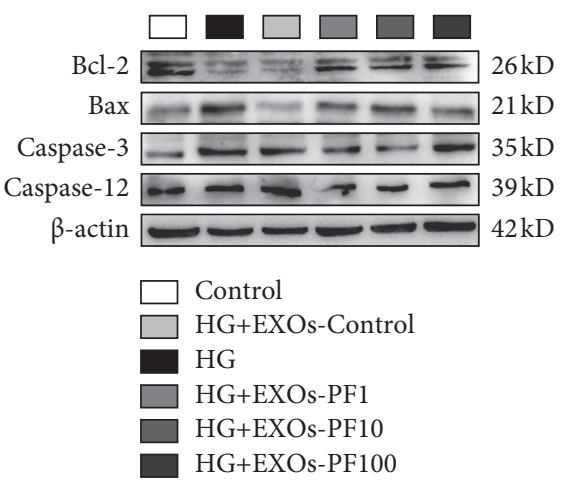

(a)

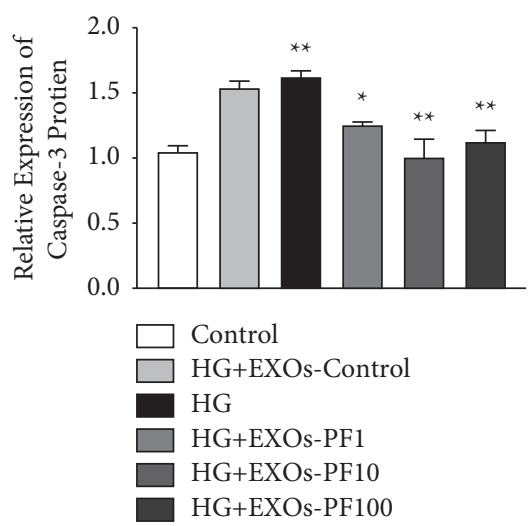

(d)

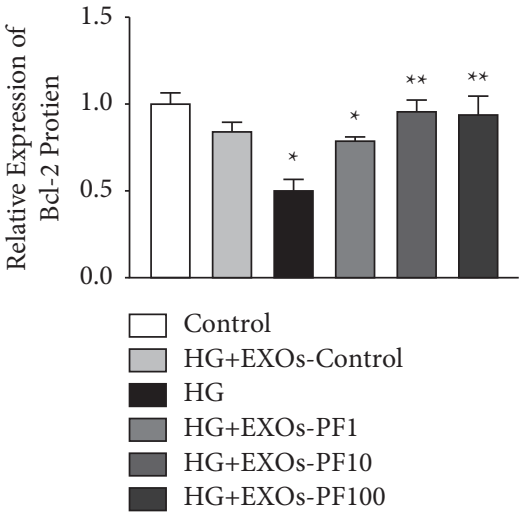

(b)

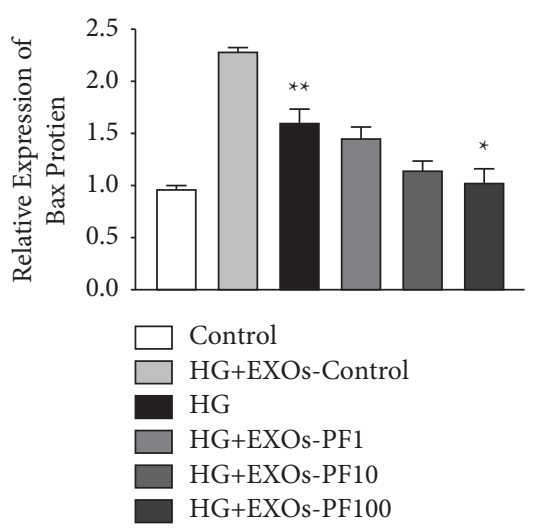

(c)

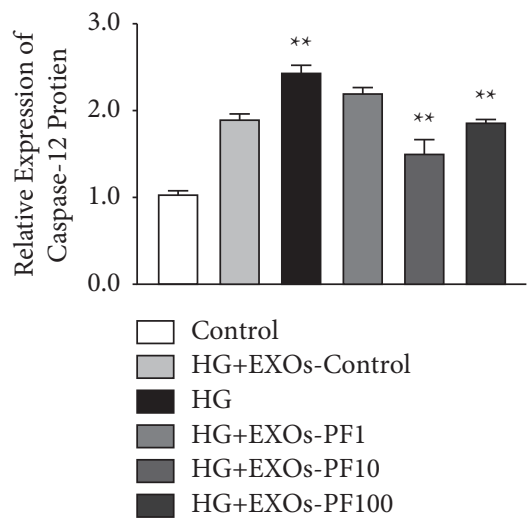

(e)

FIGURE 8: The effect of PF interfered with SC-EXOs on the apoptotic proteins in DRGn. (a) The expression of apoptotic protein in DRGn. DRGn apoptosis protein expression for (b) Bax, (c) Bcl-2, (d) Caspase-3, and (e) Caspase-12 was determined by the western blot. The results were expressed as the mean \pm standard error, $n=4$ for each group.

the expression of exosomal marker protein CD63 compared with the Control group [39], which is consistent with our experimental results. In order to eliminate the effect of other conditions on the exosomes derived from SCs, we also treated SCs with high osmotic pressure. At the same time, we tested the SCs and SC-EXOs after intervention and found 
that high osmotic pressure did not affect the activity of SCs and the information transmission between exosomes and DRGn.

The research group previously proved that DPN was characterized by demyelination caused by SCs apoptosis induced by ER stress. The pathogenesis of DPN is complicated, but ER stress is closely related to apoptosis. In the process of UPR, IRE $1 \alpha$ dissociates with GRP78 and then undergoes autophosphorylation to activate downstream reactions [40]. The results showed that both IRE1 $\alpha$ and GRP78 were expressed in exosomes, and their expression increased significantly in high glucose concentration, indicating that exosomes can carry information transmission factor and activate IRE $1 \alpha$ signal pathway. At the same time, the expression of both exosomes decreased after the intervention of PF. C/EBP homologous protein (CHOP), as a downstream protein of ER stress, is upregulated in SCs of the diabetic nephropathy rat model. Under ER stress, activated IRE $1 \alpha$ can no longer regulate the expression of transcription factors CHOP and Caspase-12 and further activate the expression of Caspase-3, aggravating apoptosis-mediated DPN damage. All these are consistent with our experimental results. CHOP played a key role in cell apoptosis induced by ER stress. It was considered to play a central role in cell apoptosis induced by ER stress. It is strongly induced by IRE1 $\alpha$ signal. Inhibiting the expression of IRE1 $\alpha$ can inhibit the expression of CHOP [41]. At the same time, in the diabetic model, the expression level of $\mathrm{XBP} 1 \mathrm{~s}$ in hippocampus decreased significantly, suggesting that the decrease in XBP-1s expression is closely related to the damage of the peripheral nervous system caused by diabetes [42]. Excessive or persistent ER stress can also activate the phosphokinase activity of IRE1 $\alpha$. Activated ASK1 induces and increases the expression of JNK by increasing the transcription of JNK. It has been found that the expression of JNK is significantly decreased after the application of IRE1 $\alpha$ phosphokinase inhibitor [43]. Our results of Western blot also show that PF interferes with SC-EXOs to affect the expression of ER stress downstream factors in DRGn, inhibits the expression of apoptotic factors Caspase-12 and Caspase-3, reduce apoptosis, and alleviates ER stress by exosomes.

In summary, our study shows that SCs can release exosomes, and the method of extracting exosomes by ultracentrifugation is feasible. Exosomes can carry ER stress initiating factor and IRE1 $\alpha$ signaling pathway information transmission factor for cell-to-cell information exchange. High glucose concentration will affect SCs secretion of exosomes, while high osmotic pressure will not affect SCs activity and exosomes secretion. PF can improve the ER stress of DRGn and affect the expression of downstream proteins such as JNK and CHOP; improve the morphology in ER of DRGn damaged by high glucose; and increase the expression of anti-apoptotic protein $\mathrm{Bcl}-2$, decrease the expression of pro-apoptotic protein Bax, inhibit the expression of Caspase family Caspase-12 and Caspase-3, and ultimately improve DPN. As a carrier of cellular communication, exosomes provide new ideas for the treatment of DPN, diabetes, and its complications.

\section{Conclusion}

This study proved that SCs can secrete exosomes. PF can affect the expression of IRE1 $\alpha$ and GRP78 which were the key proteins of IRE1 $\alpha$ signal pathway in exosomal ER stress. DRGn can absorb SC-EXOs. PF carries ER stress information by exosomes, which plays the role of anti-ER stress and reduces DRGn apoptosis, thus ameliorating DPN. Our research provided a new method for the treatment of DPN from the molecular mechanism level.

\section{Data Availability}

The data used to support the results of this study are included in the article.

\section{Conflicts of Interest}

The authors declare that they have no conflicts of interest.

\section{Acknowledgments}

This work was supported by the National Natural Science Fund Projects (Nos. 81873125 and 81473642) and the National Natural Science Fund for Youths (No. 81704014).

\section{References}

[1] P. Yan, Y. Xu, Z. Zhang et al., "Decreased plasma neuregulin 4 levels are associated with peripheral neuropathy in Chinese patients with newly diagnosed type 2 diabetes: a cross-sectional study," Cytokine, vol. 113, pp. 356-364, 2019.

[2] E. S. Kim, S. W. Lee, E. Y. Mo, S. D. Moon, and J. H. Han, "Inverse association between serum total bilirubin levels and diabetic peripheral neuropathy in patients with type 2 diabetes," Endocrine, vol. 50, no. 2, pp. 405-412, 2015.

[3] M. Kobayashi and D. W. Zochodne, "Diabetic neuropathy and the sensory neuron: new aspects of pathogenesis and their treatment implications," Journal of Diabetes Investigation, vol. 9, no. 6, pp. 1239-1254, 2018.

[4] Y. P. Liu, S. J. Shao, and H. D. Guo, "Schwann cells apoptosis is induced by high glucose in diabetic peripheral neuropathy," Life Sciences, vol. 248, Article ID 117459, 2020.

[5] H. Pan, H. Huang, L. Zhang, S. Ma, H. Yang, and H. Wang, "Adjusting internal organs and dredging channel" electroacupuncture treatment prevents the development of diabetic peripheral neuropathy by downregulating glucose-related protein 78 (GRP78) and caspase-12 in streptozotocin-diabetic rats," Journal of Diabetes, vol. 11, no. 12, pp. 928-937, 2019.

[6] W. Yao, X. Yang, J. Zhu, B. Gao, R. Liu, and L. Xu, "Tang-LuoNing, a traditional Chinese medicine, inhibits endoplasmic reticulum stress-induced apoptosis of schwann cells under high glucose environment," Evidence Based Complementary and Alternative Medicine, vol. 2017, Article ID 5193548, 9 pages, 2017.

[7] B. L. L. Clayton and B. Popko, "Endoplasmic reticulum stress and the unfolded protein response in disorders of myelinating glia," Brain Research, vol. 1648, pp. 594-602, 2016.

[8] S. S. Cao and R. J. Kaufman, "Unfolded protein response," Current Biology, vol. 22, no. 16, pp. R622-R626, 2012.

[9] D. Sharma, J. N. Singh, and S. S. Sharma, "Effects of 4-phenyl butyric acid on high glucose-induced alterations in dorsal root 
ganglion neurons," Neuroscience Letters, vol. 635, pp. 83-89, 2016.

[10] S. K. Fineberg, K. S. Kosik, and B. L. Davidson, "MicroRNAs potentiate neural development," Neuron, vol. 64, no. 3, pp. 303-309, 2009.

[11] S. Mathivanan, C. J. Fahner, G. E. Reid, and R. J. Simpson, "ExoCarta 2012: database of exosomal proteins, RNA and lipids," Nucleic Acids Research, vol. 40, pp. D1241-D1244, 2012.

[12] D. G. Phinney, M. Di Giuseppe, J. Njah et al., "Mesenchymal stem cells use extracellular vesicles to outsource mitophagy and shuttle microRNAs," Nature Communications, vol. 6, no. 1, p. 8472, 2015.

[13] N. P. Gonçalves, Y. Yan, M. Ulrichsen et al., "Modulation of small RNA signatures in schwann-cell-derived extracellular vesicles by the p75 neurotrophin receptor and sortilin," Biomedicines, vol. 8, no. 11, p. 450, 2020.

[14] R. López-Leal, F. Díaz-Viraqué, R. J. Catalán et al., "Schwann cell reprogramming into repair cells increases miRNA-21 expression in exosomes promoting axonal growth," Journal of Cell Science, vol. 133, no. 12, Article ID jcs239004, 2020.

[15] K. Elmasry, R. Mohamed, I. Sharma et al., "Epigenetic modifications in hyperhomocysteinemia: potential role in diabetic retinopathy and age-related macular degeneration," Oncotarget, vol. 9, no. 16, pp. 12562-12590, 2018.

[16] V. La Marca and A. Fierabracci, "Insights into the diagnostic potential of extracellular vesicles and their miRNA signature from liquid biopsy as early biomarkers of diabetic micro/ macrovascular complications," International Journal of Molecular Sciences, vol. 18, no. 9, p. 1974, 2017.

[17] X. J. Ning, X. H. Lu, J. C. Luo et al., "Molecular mechanism of microRNA-21 promoting schwann cell proliferation and axon regeneration during injured nerve repair," RNA Biology, vol. 17, no. 10, pp. 1508-1519, 2020.

[18] S. Kanemoto, R. Nitani, T. Murakami et al., "Multivesicular body formation enhancement and exosome release during endoplasmic reticulum stress," Biochemical and Biophysical Research Communications, vol. 480, no. 2, pp. 166-172, 2016.

[19] E. Kakazu, A. S. Mauer, M. Yin, and H. Malhi, "Hepatocytes release ceramide-enriched pro-inflammatory extracellular vesicles in an IRE1 $\alpha$-dependent manner," Journal of Lipid Research, vol. 57, no. 2, pp. 233-245, 2016.

[20] D. Dasgupta, Y. Nakao, A. S. Mauer et al., "IRE1A stimulates hepatocyte-derived extracellular vesicles that promote inflammation in mice with steatohepatitis," Gastroenterology, vol. 159, no. 4, pp. 1487-1503, 2020.

[21] J. S. Wang, Y. Huang, S. Zhang et al., "A protective role of paeoniflorin in fluctuant hyperglycemia-induced vascular endothelial injuries through antioxidative and anti-inflammatory effects and reduction of $\mathrm{PKC} \beta 1$," Oxidative Medicine and Cellular Longevity, vol. 2019, Article ID 5647219, 11 pages, 2019.

[22] X. Zhu, K. Wang, F. Zhou, and L. Zhu, "Paeoniflorin attenuates atRAL-induced oxidative stress, mitochondrial dysfunction and endoplasmic reticulum stress in retinal pigment epithelial cells via triggering Ca2+/CaMKII-dependent activation of AMPK," Archives Pharmacal Reserach, vol. 41, no. 10, pp. 1009-1018, 2018.

[23] J. Chen, M. Zhang, M. Zhu et al., "Paeoniflorin prevents endoplasmic reticulum stress-associated inflammation in lipopolysaccharide-stimulated human umbilical vein endothelial cells via the IRE $1 \alpha / \mathrm{NF}-\kappa \mathrm{B}$ signaling pathway," Food \& Function, vol. 9, no. 4, pp. 2386-2397, 2018.
[24] J. Chen, X. F. Hou, G. Wang et al., "Terpene glycoside component from moutan cortex ameliorates diabetic nephropathy by regulating endoplasmic reticulum stress-related inflammatory responses," Journal of Ethnopharmacology, vol. 193, pp. 433-444, 2016.

[25] X. L. Wang, L. W. Cui, Z. Liu et al., "Effects of TRPA1 activation and inhibition on TRPA1 and CGRP expression in dorsal root ganglion neurons," Neural Regeneration Research, vol. 14, no. 1, pp. 140-148, 2019.

[26] X. B. Wang, W. Ma, T. Luo et al., "A novel primary culture method for high-purity satellite glial cells derived from rat dorsal root ganglion," Neural Regeneration Research, vol. 14, no. 2, pp. 339-345, 2019.

[27] Z. Wu, P. Pu, Z. Su, X. Zhang, L. Nie, and Y. Chang, "Schwann cell-derived exosomes promote bone regeneration and repair by enhancing the biological activity of porous Ti6Al4V scaffolds," Biochemical and Biophysical Research Communications, vol. 531, no. 4, pp. 559-565, 2020.

[28] R. Iurlaro and C. Muñoz-Pinedo, "Cell death induced by endoplasmic reticulum stress," The FEBS Journal, vol. 283, no. 14, pp. 2640-2652, 2016.

[29] H. Dong, N. M. Adams, Y. Xu et al., “The IRE1 endoplasmic reticulum stress sensor activates natural killer cell immunity in part by regulating c-Myc," Nature Immunology, vol. 20, no. 7, pp. 865-878, 2019.

[30] L. Wang, M. Chopp, A. Szalad et al., "Exosomes derived from schwann cells ameliorate peripheral neuropathy in type 2 diabetic mice," Diabetes, vol. 69, no. 4, pp. 749-759, 2020.

[31] M. Zhang, Inam-U-Llah, X. Shi et al., "Taurine promotes neuritic growth of dorsal root ganglion cells exposed to high glucose in vitro," Advances in Experimental Medicine and Biology, vol. 1155, pp. 923-934, 2019.

[32] M. Hu, L. Hong, C. Liu et al., "Electrical stimulation enhances neuronal cell activity mediated by Schwann cell derived exosomes," Scientific Reports, vol. 9, no. 1, p. 4206, 2019.

[33] I. Allodi, E. Udina, and X. Navarro, "Specificity of peripheral nerve regeneration: interactions at the axon level," Progress in Neurobiology, vol. 98, no. 1, pp. 16-37, 2012.

[34] L. Jia, M. Chopp, L. Wang et al., "MiR-34a regulates axonal growth of dorsal root ganglia neurons by targeting FOXP2 and VAT1 in postnatal and adult mouse," Molecular Neurobiology, vol. 55, no. 12, pp. 9089-9099, 2018.

[35] E. L. Feldman, K. A. Nave, T. S. Jensen, and D. L. H. Bennett, "New horizons in diabetic neuropathy: mechanisms, bioenergetics, and pain," Neuron, vol. 93, no. 6, pp. 1296-1313, 2017.

[36] M. Zhou, M. Hu, S. He et al., "Effects of RSC96 schwann cellderived exosomes on proliferation, senescence, and apoptosis of dorsal root ganglion cells in vitro," Medical Science Monitor: International Medical Journal of Experimental and Clinical Research, vol. 24, pp. 7841-7849, 2018.

[37] A. da Silva Novaes, F. T. Borges, E. Maquigussa, V. A. Varela, M. V. S. Dias, and M. A. Boim, "Influence of high glucose on mesangial cell-derived exosome composition, secretion and cell communication," Scientific Reports, vol. 9, no. 1, p. 6270, 2019.

[38] R. Lopez-Leal and F. A. Court, "Schwann cell exosomes mediate neuron-glia communication and enhance axonal regeneration," Cellular and Molecular Neurobiology, vol. 36, no. 3, pp. 429-436, 2016.

[39] S. Han, X. Li, X. Yang, J. Zhu, Y. Zhu, and L. Xu, "Effect of paeoniflorin on apoptosis of dorsal root neurons induced by schwann cell exosomes," Journal of Medical Research, vol. 49, no. 11, pp. 49-53, 2020. 
[40] M. C. Kopp, P. R. Nowak, N. Larburu, C. J. Adams, and M. M. Ali, "In vitro FRET analysis of IRE1 and BiP association and dissociation upon endoplasmic reticulum stress," Elife, vol. 7, Article ID e30257, 2018.

[41] W. Yao, X. Yang, J. Zhu, B. Gao, H. Shi, and L. Xu, "IRE1 $\alpha$ siRNA relieves endoplasmic reticulum stress-induced apoptosis and alleviates diabetic peripheral neuropathy in vivo and in vitro," Scientific Reports, vol. 8, no. 1, p. 2579, 2018.

[42] J. Yang, X. Wu, X. Wu et al., "The multiple roles of XBP1 in regulation of glucose and lipid metabolism," Current Protein \& Peptide Science, vol. 18, no. 6, pp. 630-635, 2017.

[43] S. Yi, K. Chen, L. Zhang et al., "Endoplasmic reticulum stress is involved in stress-induced hypothalamic neuronal injury in rats via the PERK-ATF4-CHOP and IRE1-ASK1-JNK pathways," Frontiers in Cellular Neuroscience, vol. 13, p. 190, 2019. 